\title{
ANSIEDADE E DEPRESSÃO NA UNIVERSIDADE: CONTRIBUIÇÕES DA TERAPIA COMUNITÁRIA INTEGRATIVA
}

\author{
ANSIEDAD Y DEPRESIÓN EN LA UNIVERSIDAD: CONTRIBUCIONES DE LA \\ TERAPIA COMUNITARIA INTEGRATIVA
}

\author{
ANXIETY AND DEPRESSION IN THE UNIVERSITY: CONTRIBUTIONS \\ FROM INTEGRATIVE COMMUNITY THERAPY
}

\author{
Jucelei Pascoal BOARETTO ${ }^{1}$ \\ Milene Zanoni da SILVA ${ }^{2}$
}

Eleine Aparecida Penha MARTINS ${ }^{3}$

RESUMO: Este artigo avalia os níveis de ansiedade e depressão dos estudantes de graduação e pós-graduação de universidade pública no sul do país antes e após participação em Terapia Comunitária Integrativa. Utilizamos a pesquisa experimental, com desenho pré/pós-teste com intervenção da Terapia Comunitária Integrativa, desenvolvida em 2018/2019, com uso da Escala de "Hospital Anxiety and Depression" e análise realizada por programa estatístico R Core Team e Teste de Wilcoxon. Pode-se observar diminuição estatisticamente significativa dos escores de provável ansiedade e depressão em aproximadamente $30 \%$ dos estudantes da graduação como para pós-graduação, o que traz o potencial da terapia comunitária integrativa para manejo destas patologias além de promover a saúde, autoconhecimento, fortalecimento de vínculos. A Terapia Comunitária Integrativa diminuiu os níveis de ansiedade e de depressão nos estudantes. Desse modo, pode ser considerada, uma ferramenta importante para auxílio ao cuidado de saúde no ambiente estudantil universitário.

PALAVRAS-CHAVE: Terapias complementares. Universidade. Ansiedade. Depressão.

RESUMEN: Este artículo evalúa los niveles de ansiedad y depresión de estudiantes de pre y posgrado de una universidad pública del sur del país antes y después de participar en la Terapia Integrativa Comunitaria. Se utilizó la investigación experimental, con diseño pre / post test con la intervención de la Terapia Comunitaria Integrativa, desarrollada en 2018/2019, utilizando la Escala "Hospital Anxiety and Depression" y análisis realizado por el programa estadístico $R$ Core Team y Wilcoxon Test. Es posible observar una disminución estadisticamente significativa en los puntajes de probable ansiedad y depresión en aproximadamente el 30\% de los estudiantes de pre y posgrado, lo que trae el potencial de la terapia comunitaria integradora para el manejo de estas patologías además de promover la

${ }^{1}$ Universidade Estadual de Londrina (UEL), Londrina - PR - Brasil. Mestranda no Programa de Pós-Graduação em Enfermagem. Coordenadora das Práticas Integrativas e Complementares do Município de Londrina. ORCID: https://orcid.org/0000-0002-5562-9370. E-mail: juceleipb@gmail.com

${ }^{2}$ Universidade Federal do Paraná (UFR), Curitiba - PR - Brasil. Professora Adjunta II do Departamento de Saúde Coletiva. Doutorado em Saúde Coletiva (UEL). ORCID: http://orcid.org/0000-0002-1177-9668. E-mail: milenezanoni@gmail.com

${ }^{3}$ Universidade Estadual de Londrina (UEL), Londrina - PR - Brasil. Professora Associada do Departamento de Enfermagem e docente do Programa de Pós-Graduação em Enfermagem. Doutorado em Enfermagem Fundamental (EERP-USP). ORCID: https://orcid.org/0000-0001-6649-9340. E-mail: eleinemartins@gmail.com 
salud, el autoconocimiento, el fortalecimiento. de bonos. La Terapia Integrativa Comunitaria disminuyó los niveles de ansiedad y depresión en los estudiantes. Por lo tanto, puede considerarse una herramienta importante para ayudar a la atención de la salud en el entorno del estudiante universitario.

PALABRAS CLAVE: Terapias complementarias. Universidad. Ansiedad. Depresión.

ABSTRACT: This paper assesses the levels of anxiety and depression of undergraduate and postgraduate students at public universities in the south of the country before and after participation in Integrative Community Therapy. We use the experimental research, with pre/post-test design with Integrative Community Therapy intervention, developed in 2018/2019, using the "Hospital Anxiety and Depression" Scale and analysis performed by statistical program $R$ Core Team and Wilcoxon Test. It can be observed a statistically significant decrease of the probable anxiety and depression scores in approximately $30 \%$ of undergraduate and postgraduate students, which brings the potential of integrative community therapy to manage these pathologies besides promoting health, self-knowledge, strengthening bonds. Integrative Community Therapy has decreased the levels of anxiety and depression in students. Therefore, it can be considered an important tool to help health care in the university student environment.

KEYWORDS: Complementary therapies. University. Anxiety. Depression.

\section{Introdução}

No ano de 2017, um importante relatório da Organização Mundial da Saúde (OMS), apontou que aproximadamente 450 milhões de pessoas sofriam de algum tipo de perturbação mental ou neurobiológica e, o Brasil, seria o país mais ansioso e deprimido da América Latina (OMS, 2017).

Este fato tornou-se um desafio tanto para a população nacional como para os profissionais da área da saúde, principalmente no que se refere ao preparo para atuar nesta área. Estudo realizado numa instituição de ensino superior do Nordeste do Brasil encontrou prevalência de ansiedade e depressão entre estudantes dos cursos da saúde de 36,1\% e 28,6\%, respectivamente (LEÃO et al., 2018).

Dificuldade de adaptação e mudança na rotina dos estudantes que adentram no ensino superior são evidentes e geram, praticamente, em 50\% desta população, transtornos decorridos da ansiedade. Dessa maneira, Medeiros e Bittencourt (MEDEIROS; BITTENCOUR, 2017), apontam que algumas medidas devem ser realizadas, antes que a ansiedade se torne maléfica, ou seja, patológica.

O uso de medicamentos psicotrópicos, tal como antidepressivos e benzodiazepínicos, tem sido a principal estratégia terapêutica para tratamento de sofrimento difuso e transtornos 
mentais no Brasil e este consumo vem aumentando, por vezes de forma desnecessária e irracional (WAGNER, 2015).

Considerando tal problemática, torna-se importante estabelecer outras ofertas terapêuticas nos sistemas de saúde, que extrapolam o modelo biomédico (SOUSA et al., 2017). Com este intuito, em 2006, o Ministério da Saúde (MS) aprovou a Política Nacional de Práticas Integrativas e Complementares (PNPIC) (BRASIL, 2018) com inserção de 5 práticas integrativas e, em 2017 e 2018, foram aprovadas mais 24 PIC no SUS, entre elas, a Terapia Comunitária Integrativa (TCI), que é uma PIC genuinamente brasileira, classificada pelo Ministério da Saúde como uma abordagem psicossocial avançada (BRASIL, 2018), cuja abordagem é grupal, criada em 1987, pelo Professor Dr. Adalberto Barreto, que proporciona um espaço de acolhimento e partilha de sentimentos e experiências de vida, onde recursos e competências das pessoas são mobilizados por meio da ação terapêutica do próprio grupo (BARRETO, 2010). Sendo um recurso terapêutico não-convencional, a TCI é uma política pública viável, de baixo custo e não medicalizadora, que objetiva não apenas prevenção e tratamento dos transtornos mentais, mas também promove a saúde mental, numa perspectiva positiva e integral (SILVA, 2015).

Logo, avaliar a TCI no manejo da ansiedade e da depressão dentro do ambiente acadêmico é de suma importância, pois ela contribui para construção de redes de apoio social, o que se traduz em mudanças e no reconhecimento das próprias competências (JATAI; SILVA, 2012). Justifica-se esse estudo, pois, pela revisão de literatura realizada, não foi identificado estudo que fizesse a avaliação entre os níveis de ansiedade e de depressão e a prática da TCI. Além disso, é importante a produção de evidências científicas acerca do impacto desta PIC na saúde humana, para sua manutenção e expansão como política pública no SUS.

Diante do exposto, objetivou-se avaliar os efeitos da prática da Terapia Comunitária Integrativa nos níveis de ansiedade e de depressão nos estudantes de graduação e pós-graduação de uma universidade pública no sul do Brasil antes e após a participação em rodas de TCI.

\section{Método}

Trata-se de uma pesquisa quase-experimental, com desenho pré/pós-teste. A tipologia de estudos quase-experimentais não possui distribuição aleatória dos entrevistados pelos tratamentos e nem grupos-controle. Possibilita a observação de como, quando e a quem ocorrem as relações de causa-efeito, sem um experimento (FERREIRA NETO, 2015). 
Adotou-se como critério de inclusão para esta pesquisa, estudantes matriculados nos cursos de graduação em enfermagem, farmácia e fisioterapia e no Mestrado em Enfermagem do Centro de Ciências da Saúde de uma universidade estadual pública, com no mínimo 12 meses de frequência nas aulas e que, aceitaram o convite realizado via e-mail ao centro acadêmico e aos colegiados de cursos, trabalhando então, com uma amostragem por conveniência. Em decorrência da metodologia requerer que a pessoa esteja empaticamente disposta a compartilhar suas histórias de vida, a amostragem probabilística não intencional não se aplica neste tipo de intervenção. Foram excluídos os estudantes que não participaram de todos os encontros programados, bem como, aqueles que não responderam ao $e$-mail enviado.

Foram realizados 5 encontros, em cada grupo de estudantes, no período de agosto de 2018 a abril de 2019, totalizando 20 rodas de TCI. A coleta de dados das variáveis independentes (sociodemográficas) e dependentes (depressão e ansiedade) ocorreu em 2 momentos, antes (T0) e depois (T5) da intervenção. A intervenção de TCI realizada seguiu a técnica segundo Barreto (2008), seguindo as etapas de acolhimento, a escolha do tema, a contextualização, a problematização, a conclusão e a apreciação (BARRETO, 2012) com terapeuta comunitária com expertise na metodologia.

O instrumento utilizado para avaliar os níveis de ansiedade e depressão foi a Escala de “Hospital Anxiety and Depression” (ZIGMOND; SNAITH, 1983). Tal escala foi validada para o português por Botega (BOTEGA et al., 1995) e colaboradores em 1995 no Brasil e, é constituída por 14 questões de múltipla escolha, contendo duas "subescalas", ou seja, sete itens para as questões ímpares $(1,3,5,7,9,11,13)$, voltadas aos níveis de ansiedade e, sete itens para as questões pares $(2,4,6,8,10,12,14)$, voltadas aos níveis de depressão. A pontuação global em cada "subescala" varia de zero até 21 pontos, sendo que de 0 a 7 pontos indica resultado improvável; de oito a 11 pontos o resultado é possível, e de 12 a 21 pontos o resultado é provável, ou seja, o indivíduo pode ser classificado com nível de ansiedade e/ou depressão. É um instrumento de alta sensibilidade na detecção dos níveis ansiedade e/ou depressão (CASTRO et al., 2006) e, não objetiva atribuir um diagnóstico clínico de transtornos mentais, mas sim, fornecer subsídios para que os serviços avaliassem os níveis de ansiedade e depressão e buscassem estratégias de prevenção (ZIGMOND; SNAITH, 1983).

Os dados obtidos foram tabulados e duplamente digitados em planilha do Excel e importados e analisados no programa $R$ Core Team, versão 2017. Foram construídas tabelas de distribuição de frequência das variáveis estudadas, calculando-se as medianas para as variáveis contínuas de distribuição não normal. Para o cálculo de associação entre as variáveis categóricas de exposição e de desfecho foi realizada, inicialmente, a análise para os estudantes cujos dados 
atenderam aos pressupostos de Normalidade e Homogeneidade de Variâncias, tendo sido realizado o teste T de Student (MESSETTI, 2018).

Para os estudantes com dados que não atenderam aos pressupostos, foi realizado o teste equivalente não paramétrico de Wilcoxon. Para ambos os testes, foram escolhidas as amostras do tipo pareada. Considerou-se p-valor $<0,05$ como significativo (GOESSLER, 2018).

Foram atendidos os preceitos éticos estabelecidos na Resolução $n^{\circ}$ 466/2012 do Conselho Nacional de Saúde e a pesquisa foi aprovada pelo Comitê de Ética em Pesquisa, segundo parecer $\mathrm{n}^{\circ}$ 2.682.912. Todos os estudantes consentiram a participação por meio do Termo de Consentimento Livre e Esclarecido.

\section{Resultados}

O estudo totalizou 25 estudantes que cumpriram com os critérios de inclusão, ou seja, que participaram das cinco rodas de TCI.

Quanto ao perfil sociodemográfico da população estudada, $84 \%(n=21)$ das estudantes eram mulheres e todos os estudantes se enquadraram na faixa etária de adultos (19-29 anos).

Foram constituídos quatro grupos, sendo que $28 \%$ dos componentes eram estudantes da pós-graduação e $72 \%$ de estudantes da graduação que formaram três grupos distintos, sendo enfermagem (20\%), fisioterapia (32\%) e farmácia (20\%). A tabela 1 apresenta as prevalências de ansiedade (52\%) e depressão (12\%) da população estudada. Com relação à ansiedade, o curso com maior escore foi fisioterapia (24\%). No caso da depressão, a taxa foi de $4 \%$ entre os cursos de graduação (dados não presentes em tabela). Após a intervenção com a Terapia Comunitária Integrativa os níveis de escore de "provável" ansiedade reduziram de 52\% para $24 \%$ ( $<<0,05)$. Tal como também ocorreu com a depressão com diminuição de $12 \%$ para $4 \%$ $(\mathrm{p}<0,05)$. 
Tabela 1 - Escores de provável ansiedade/depressão por sexo da Escala HAD dos estudantes antes e depois de participarem das rodas de Terapia Comunitária Integrativa - Londrina - PR 2019

\begin{tabular}{llll}
\hline & Antes & Depois & $\mathrm{p}$ \\
\hline Total & $\mathbf{2 5 ( 1 0 0 )}$ & $\mathbf{2 5}(\mathbf{1 0 0 )}$ & \\
Feminino & $21(84)$ & $21(84)$ & \\
Masculino & $4(16)$ & $4(16)$ & \\
Ansiedade & $\mathbf{1 3 ( 5 2 )}$ & $\mathbf{6}(\mathbf{2 4 \% )}$ & $<\mathbf{0 , 0 5}$ \\
Feminino & $12(48)$ & $5(20)$ & \\
Masculino & $1(4)$ & $1(4)$ & \\
Depressão & $\mathbf{3 ( 1 2 )}$ & $\mathbf{1 ( 4 \% )}$ & $\mathbf{< 0 , 0 5}$ \\
Feminino & $2(8)$ & $0(0)$ & \\
Masculino & $1(4)$ & $1(4$ & \\
\hline
\end{tabular}

Fonte: Dados da pesquisa (2018-2019)

Na figura 1, ao comparar a taxa de ansiedade antes e após as rodas de TCI observou-se uma redução estatisticamente significativa na mediana de $30 \%$ nos escores do HAD de ansiedade $(p=0,0000078)$ e depressão $(0,0009)$.

Figura 1 - Comparação dos níveis do escore "provável" para ansiedade e depressão antes e após a prática da Terapia Comunitária Integrativa com estudantes dos cursos de graduação e pós-graduação. Londrina-PR, 2018-2019
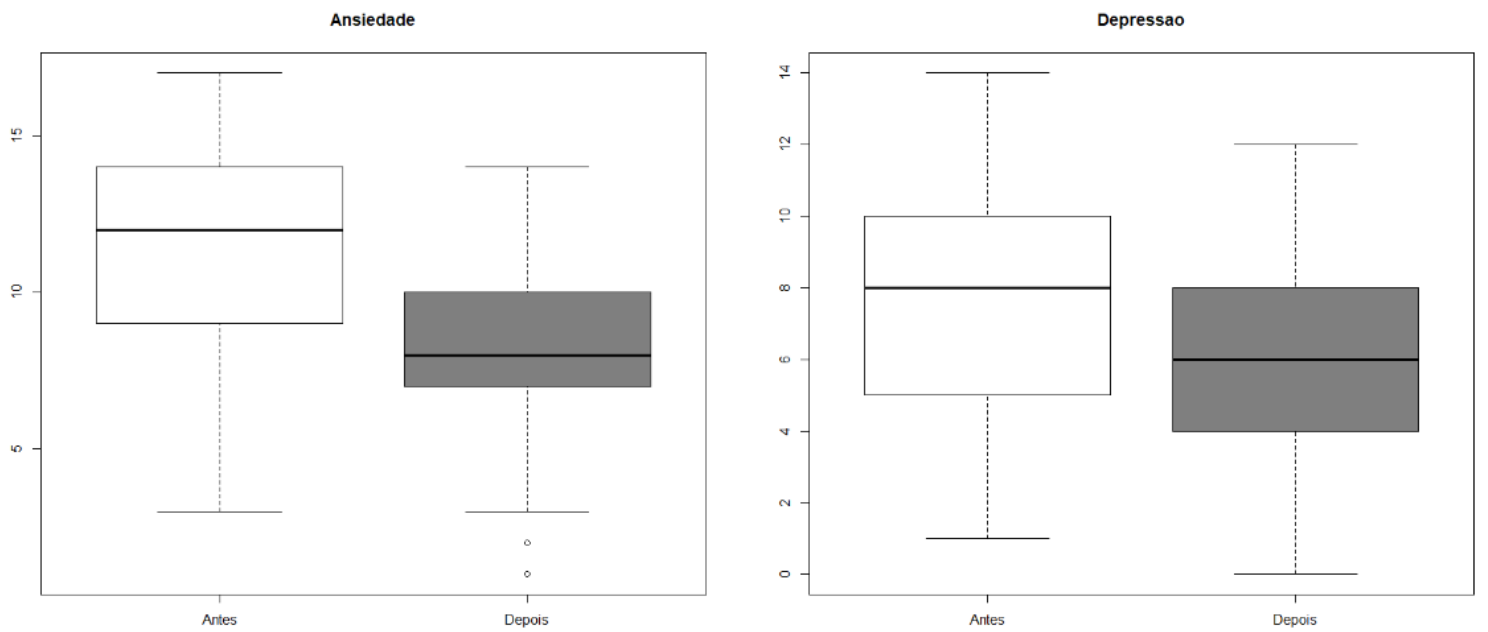

Fonte: Dados da pesquisa (2018-2019) 
Tabela 2 - Comparação da prevalência de Escores de Ansiedade antes e depois da prática da Terapia Comunitária Integrativa com estudantes dos cursos de graduação e pós-graduação.

Londrina-PR, 2019

\begin{tabular}{lccc|ccc}
\hline \multirow{2}{*}{ Cursos } & \multicolumn{2}{c|}{ \% Escore para Ansiedade T0 } & \multicolumn{3}{c}{ \% Escore para Ansiedade T1 } \\
\cline { 2 - 7 } & Improvável & Possível & Provável & Improvável & Possível & Provável \\
\hline EM (1) & 0 & 43 & 57 & 43 & 14 & 43 \\
E (2) & 0 & 80 & 20 & 60 & 20 & 20 \\
Fs (3) & 12,5 & 12,5 & 75 & 50 & 37,5 & 12,5 \\
Fa (4) & 0 & 40 & 60 & 40 & 40 & 20 \\
\hline
\end{tabular}

(1) EM - Estudantes da Pós Graduação: Mestrado em enfermagem

(2) E - Estudantes da Graduação em Enfermagem

(3) Fs -Estudantes da Graduação em Fisioterapia

(4) Fa-Estudantes da Graduação em Farmácia

Fonte: Dados da pesquisa (2018-2019)

Nas tabelas 2 e 3 é possível verificar as taxas de escores de ansiedade e depressão antes e após a intervenção de Terapia Comunitária Integrativa. Ao se considerar os níveis de ansiedade no $\mathrm{T} 0$ e $\mathrm{T} 1$, todos os cursos obtiveram redução da prevalência na categoria "provável”, com exceção da enfermagem (tabela 2). A fisioterapia teve o melhor percentual de diminuição, passando de 75\% no T0 para 12,5\% no T1. Quanto à depressão, houve redução da categoria "provável” nos cursos de farmácia e enfermagem.

Tabela 3 - Comparação da prevalência de Escores de depressão antes e depois da prática da Terapia Comunitária Integrativa com estudantes dos cursos de graduação e pós-graduação.

Londrina-PR, 2019

\begin{tabular}{lccc|ccc}
\hline \multirow{2}{*}{ Cursos } & \multicolumn{3}{c|}{ \% Escore para Depressão T0 } & \multicolumn{3}{c}{ \% Escore para Depressão T1 } \\
\cline { 2 - 7 } & Improvável & Possível & Provável & Improvável & Possível & Provável \\
\hline EM (1) & 57 & 43 & 0 & 57 & 43 & 0 \\
E (2) & 40 & 40 & 20 & 40 & 60 & 0 \\
Fs (3) & 37,5 & 50 & 12,5 & 62,5 & 25 & 12,5 \\
Fa (4) & 20 & 60 & 20 & 100 & 0 & 0 \\
\hline
\end{tabular}

(1) EM - Estudantes da Pós Graduação: Mestrado em enfermagem

(2) $\mathbf{E}$ - Estudantes da Graduação em Enfermagem

(3) Fs -Estudantes da Graduação em Fisioterapia

(4) Fa-Estudantes da Graduação em Farmácia

Fonte: Dados da pesquisa (2018-2019) 
Figura 2 - Distribuição dos níveis de provável ansiedade antes e após as cinco rodas de Terapia Comunitária Integrativa com estudantes dos cursos de graduação e pós-graduação. Londrina-PR, 2018-2019

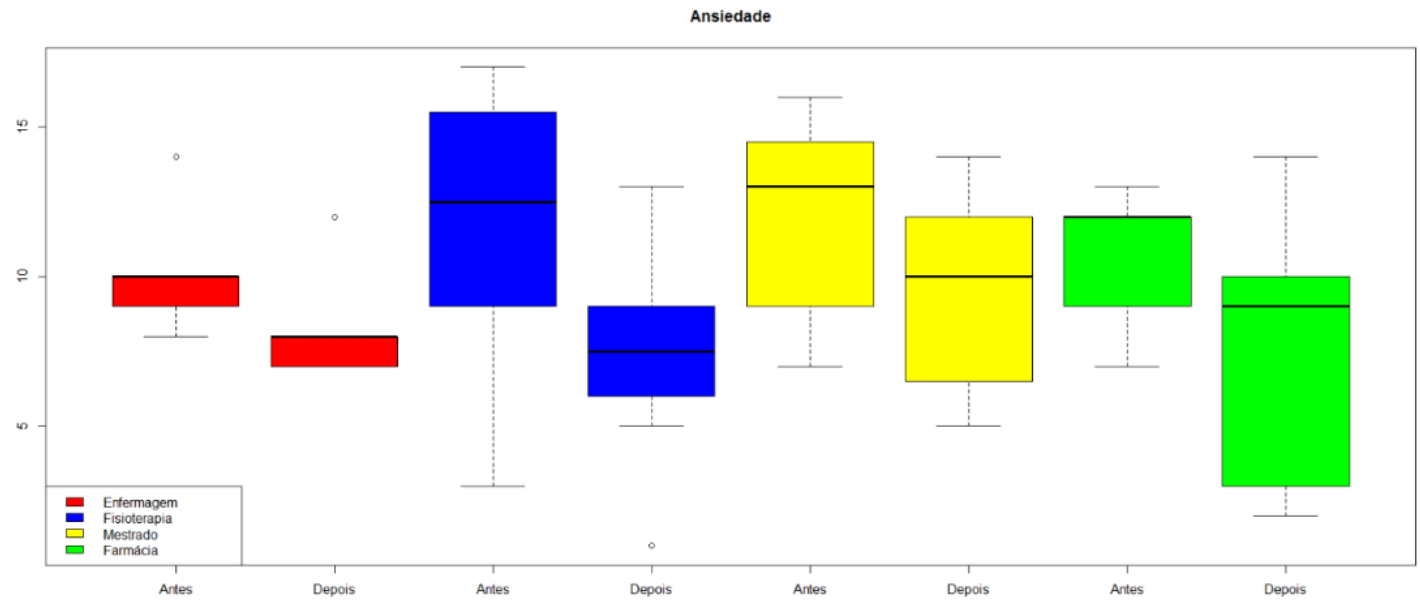

Fonte: Dados da pesquisa (2018-2019)

A figura 2 demonstra as medianas dos escores de ansiedade entre os estudantes dos três cursos de graduação em enfermagem, fisioterapia, farmácia e de pós-graduação do mestrado em enfermagem. Apresentaram resultados estatisticamente significativos com $p<0,05$, sendo que os que obtiveram a maior melhora foram os da farmácia.

Analisando os dados apresentados na figura 3, a qual traz a distribuição das medianas dos escores de depressão antes e após a participação na TCI, infere-se que, os níveis de depressão diminuíram em todos os estudantes que participaram da TCI, tendo uma melhora expressiva para os estudantes de farmácia $(\mathrm{p}=0,02724)$.

Figura 3 - Distribuição dos níveis de provável depressão antes e após as cinco rodas de Terapia Comunitária Integrativa com estudantes dos cursos de graduação e pós-graduação. Londrina-PR, 2018-2019

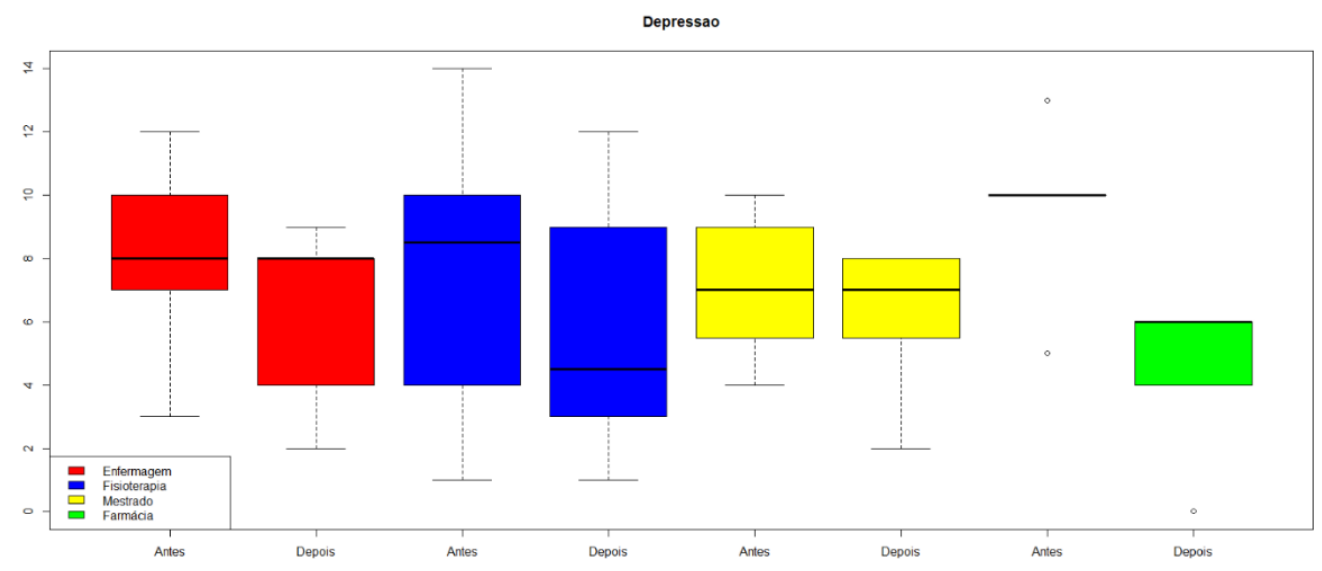

Fonte: Dados da pesquisa (2018-2019) 
Os escores de ansiedade e depressão diminuíram, o que ficou evidente ao final do estudo, pois os estudantes apontaram redução da "tensão" considerando que apenas $8 \%$ referiram, ao final, a alternativa "nunca". Em relação ao "medo", os resultados apontaram uma queda de $24 \%$ entre os estudantes que se consideravam "sim, de jeito muito forte" e um aumento no percentual de "sim, mas não tão forte" de 8\%. Houve também uma redução referente ao sentir-se "inquieto," "preocupado", além da melhora em "se sentir relaxado", conforme dados analisados na comparação dos instrumentos aplicados antes e depois das cinco rodas de TCI.

Nos índices relacionados à depressão, o quesito "alegria”, "dar risada e se divertir", "gostar das mesmas coisas que antes", "ânimo” e "interesse em cuidar da própria aparência" apresentaram melhora nas frequências no pós teste (T1) em comparação ao pré teste (T0). Já o "ânimo" e o "interesse em cuidar da própria aparência” também mostraram melhora.

\section{Discussão}

No presente estudo pode-se verificar que mais da metade dos participantes da pesquisa estavam com escores de provável ansiedade (52\%) e 12\% com escores de depressão. Além disso, a terapia comunitária se mostrou uma estratégia relevante para o manejo em saúde mental na formação superior, considerando que houve uma diminuição da ansiedade e depressão em aproximadamente $30 \%$ tanto para os estudantes da graduação como para os da pós graduação nos diferentes cursos da área da saúde. Em concordância com o estudo de Almeida (2015), comprova-se a necessidade de utilizar instrumentos e estratégias para a promoção da saúde mental dos estudantes universitários.

Corroborando para esse entendimento, estudo realizado por Lantyer et al. (2016) reafirmam a importância do desenvolvimento de programas de intervenção psicoterápica na universidade. Svoboda et al. (2018) igualmente enfatizam que uma das intervenções possíveis para reduzir o estresse constitui-se nas rodas de TCI, utilizadas em duas universidades brasileiras em 2017, apontando para 62,5\%, dos encontros sobre o tema "estresse" (SVOBODA et al., 2018).

A metodologia utilizada neste estudo visa resgatar a autoestima dos participantes, trazendo à luz da consciência dos mesmos que, se existe uma dificuldade na vida de cada um, também existem dentro deles, os mecanismos capazes de superá-la e transpô-la, conseguindo a superação (BARRETO, 2008), e não, diagnosticar nenhum participante como patologicamente 
ansioso ou depressivo, ou seja, a escala HAD, permite a análise quantitativa dos níveis de ansiedade e de depressão (PETERMANN, 2015). Este mesmo instrumento, teve comprovada a sua eficácia em diferentes artigos e contextos, como demonstra os estudos de Terol-Cantero e Cabrera-Perona (2015) e Faro (2015).

$\mathrm{Na}$ análise geral do instrumento utilizado no pré e no pós teste para avaliar o efeito da TCI, observou-se que os estudantes diminuíram a "tensão”, o "medo" e a "preocupação”, além de referirem melhora no quesito de "se sentir relaxado", o que possibilita a mensuração quantitativa dos benefícios pela participação nas rodas de TCI.

Segundo Cruz et al. (2019), em um estudo realizado em uma universidade do interior do estado de São Paulo mostraram que a TCI foi citada como estratégia de apoio aos estudantes, ressignificando suas vidas a partir da partilha e aprendendo a realizar uma autoanálise, individualmente.

Estes dados corroboram para a análise do instrumento aplicado no pré e no pós teste neste estudo, o qual demonstrou uma efetiva diminuição de sintomas negativos e o aumento dos sintomas positivos, diminuindo os índices de ansiedade e de depressão entre os estudantes participantes do estudo.

O instrumento também aborda questões voltadas ao sentir-se "inquieto", o qual diminuiu 50\% entre os participantes, apontando para a necessidade de espaços de partilha, acolhimento e escuta entre os universitários de cursos da saúde, visando o desenvolvimento pessoal, além do acadêmico (ARAÚJO; BRESSAM, 2017).

No que tange à depressão, as subescalas que quantificaram os níveis dos estudantes, tiveram uma pontuação positiva no quesito “alegria”, bem como discretas mudanças, porém significativas, no quesito "dar risada e se divertir" e "gostar das mesmas coisas que antes", o que demonstra a pertença dessas pessoas em relação a sua identidade e percepção pessoal e a necessidade de estratégias que promovem o cuidado de forma singular, ou seja, na individualidade de cada ser humano (SOUZA et al., 2017).

$\mathrm{O}$ ânimo e o interesse em cuidar da própria aparência também mostraram melhora expressiva após a participação nas rodas de TCI, bem como o fato dos estudantes conseguirem sentir "prazer" quando assistem a um bom programa de televisão, de rádio ou quando leem alguma coisa, pois as rodas de TCI trabalham os sentimentos de forma acolhedora e participativa, promovendo o empoderamento pessoal e o autoconhecimento (BOARETTO; MARTINS, 2019).

Considera-se importante a participação do estudante em um programa em que, durante cada encontro, ele pode perceber que tudo aquilo que lhe fazia mal, era passível de resolução 
por ele mesmo, pois ao ouvir os demais colegas do grupo, identificou que, existiam "emoções/sentimentos" dentro dele mesmo que deveriam ser modificados. Dessa forma, as emoções/sentimentos são invisíveis na maioria das vezes e, mesmo sendo alvo de estudos acadêmicos nas áreas de psicologia, medicina e/ou enfermagem, ainda existe a necessidade de promover o cuidado antes que as patologias aconteçam, pois quanto maior a qualidade de vida, menores serão os índices de ansiedade e de depressão (BORINE et al., 2015).

A estratégia da TCI leva em consideração que as demandas dos cursos da saúde são extensas (em grade integral), o que exige do universitário maior tempo fora de seu ambiente de convívio familiar e social, com uma mudança em seu estilo de vida; acresce-se que há elevação da carga de responsabilidades e exigências, o que pode acarretar distúrbios emocionais e psíquicos, estudados dentro da psicologia (SOUZA et al., 2017).

O sofrimento humano, apesar de ser tema de diversas pesquisas e palestras no mundo todo, ainda precisa ser acolhido e ressignificado, tal como é a proposta trazida pela TCI. Com a ampliação das novas formas de cuidar em saúde, observa-se um novo olhar sobre as práticas desempenhadas em outras áreas da saúde. Empiricamente reconheceu-se o valor da TCI, pois os estudantes apresentaram melhora dos níveis de ansiedade e de depressão.

Segundo Faro (2015), a ansiedade e a depressão são as maiores causas de incapacidade no mundo, estando associadas às diferentes patologias incapacitantes: doenças cardíacas, mortes prematuras e distintos tipos de câncer. Somente quando os indivíduos reconhecerem a necessidade de buscar estratégias para reduzir esses sentimentos podem evitar os riscos para o seu desenvolvimento. A ansiedade e/ou a depressão são apontadas, em diferentes estudos, como fatores presentes em diversas instituições de ensino superior (CASTRO, 2017).

Neste estudo, os cuidados metodológicos para garantir a validade interna da pesquisa envolveram a realização das rodas de TCI por terapeuta comunitária com expertise, a coleta de dados no T0 e T1 no momento adequado (início e após 5 encontros), sem lacunas de tempo e os critérios de inclusão envolveram a análise apenas dos estudantes que participaram de todos os encontros. A amostragem intencional que poderia ser um limitante, contudo neste tipo de intervenção não é possível a realização de amostragem probabilística. Portanto, as desvantagens deste tipo de amostragem são as típicas da amostragem não probabilística como impossibilidade de generalização (GOESSLER, 2018).

No entanto, o estudo demonstrou que, os níveis de ansiedade e de depressão, sofreram alteração, após a participação das rodas de TCI. Percebe-se uma lacuna no conhecimento voltado ao uso de práticas integrativas e complementares voltadas ao cuidado da saúde mental 
dos estudantes universitários, por isso, este estudo promove um avanço ao conhecimento e deixa o caminho aberto para novas pesquisas dentro da temática em questão.

\section{Conclusão}

Os resultados encontrados na análise do pré e do pós teste, apontam para a redução significativa dos níveis de escores de ansiedade e de depressão dos estudantes universitários, após a participação nas rodas de Terapia Comunitária Integrativa. Os quesitos "tensão”, "medo" e "preocupação", medidos pelos itens de ansiedade diminuíram e os estudantes universitários, também referiram melhora no quesito "se sentir relaxado," o que, demonstra a mensuração realizada, quantitativamente, dos benefícios de participar das rodas de Terapia Comunitária Integrativa.

Com relação a depressão, constatou-se pontuação positiva no quesito “alegria”, bem como discretas mudanças, como no quesito "dar risada e se divertir" e "gostar das mesmas coisas que antes" e uma melhora, significativa, em relação ao "ânimo" e ao "interesse em cuidar da própria aparência."

A partir dos resultados encontrados após a coleta dos dados e a análise estatística, o que já havia sido percebido durante os anos de vivência dentro desta prática integrativa e complementar da TCI pela pesquisadora, é apresentado, à luz da ciência, que essa estratégia pode ser utilizada para reduzir os níveis de ansiedade e depressão. Os resultados deste estudo demonstram, também, as possibilidades para a realização de novas pesquisas dentro desta temática ansiedade e depressão, sobrepondo as fragilidades aqui apontadas como propulsoras para novas estratégias.

\section{REFERÊNCIAS}

ALMEIDA, J. S. P. A saúde mental global, a depressão, a ansiedade e os comportamentos de risco nos estudantes do ensino superior: estudo de prevalência e correlação. Orientador: Ricardo Gusmão. 2015. 236 f. Tese (Doutorado em Ciências da Vida) - Universidade Nova de Lisboa, Lisboa, 2014. Disponível em: http://hdl.handle.net/10400.11/2939. Acesso em: 07 ago. 2020.

ARAÚJO, C. L.; BRESSAM, V. R. Ações de promoção à saúde, atenção psicossocial e educacional como práticas de integração universitária. In: Congresos CLABES, 7., 2017, Córdoba. Anais [...]. Córdoba, Argentina: Universidad Nacional de Córdoba, 2017. p. 1-8.

BARRETO, A. P. Terapia Comunitária: passo a passo. 3. ed. Revista e ampliada. Fortaleza: Gráfica LCR, 408 p., 2008. 
BARRETO, A. P.; BARRETO, M. R. Guia para a prática em Terapia Comunitária Sistêmica Integrativa. Fortaleza: Gráfica LCR, 152 p., 2010.

BOARETTO, J. P.; MARTINS, E. A. P. Avaliação da efetividade do uso da terapia comunitária e integrativa na saúde pública/Evaluation of the effectiveness of the use of community and integrative therapy in public health. Brazilian Journal of Health Review, v. 2, n. 4, p. 3385-3392, 2019.

BORINE, R. C. C. et al. Relação entre a qualidade de vida e o estresse em acadêmicos da área da saúde. Estudos Interdisciplinares em Psicologia, v. 6, n. 1, p. 100-118, 2015.

BOTEGA, N. J. et al. Transtornos do humor em enfermaria de clínica médica e validação de escala de medida (HAD) de ansiedade e depressão. Revista de Saúde Pública, v. 29, p. 359363, 1995.

BRASIL. Ministério da Saúde. Secretaria de Atenção à Saúde. Departamento de Atenção Básica. Manual de implantação de serviços de práticas integrativas e complementares no SUS. Brasília: Ministério da Saúde, 56 p., 2018a.

BRASIL. Ministério da Saúde. Secretaria de Atenção à Saúde. Departamento de Atenção Básica. Política Nacional de Práticas Integrativas e Complementares no SUS: atitude de ampliação de acesso. 2. ed. 1. reimp. Brasília: Ministério da Saúde, 96p., 2018b.

CASTRO, M. M. C. et al. Validade da escala hospitalar de ansiedade e depressão em pacientes com dor crônica. Revista Brasileira de Anestesiologia, v. 56, n. 5, jul./ago. 2006.

CASTRO, V. R. Reflexões sobre a saúde mental do estudante universitário: estudo empírico com estudantes de uma instituição pública de ensino superior. Revista Gestão em FocoEdição, p. 380-481, 2017.

CRUZ, P. L. B. et al. Transtorno mental comum entre estudantes de enfermagem e fatores envolvidos. Revista de Enfermagem do Centro Oeste Mineiro, v. 9, 2019.

FARO, A. Análise fatorial confirmatória e normatização da Hospital Anxiety and Depression Scale (HADS). Psicologia: Teoria e Pesquisa, Brasília, v. 31, n. 3, p. 349-353, jul./set. 2015. Disponível em: http://www.scielo.br/scielo.phpS0102-37722015000300349. Acesso em: 21 out. 2019.

FERREIRA NETO, J. L. Pesquisa e metodologia em Michel Foucault. Psic.: Teor. e Pesq. [online], v. 31, n. 3, p. 411-420, 2015. ISSN 0102-3772. Disponível em:

https://www.scielo.br/scielo.php?script=sci_arttext\&pid=S010237722015000300411\&lng=pt\&tlng=pt. Acesso em: 08 ago. 2020. DOI: https://doi.org/10.1590/0102-377220150321914100420

GOESSLER, K. Bioestatística: específico PPGENF. Londrina: SAUSTAT, 2018.

JATAI, J. M.; SILVA, L. M. S. Enfermagem e a implantação da Terapia Comunitária Integrativa na Estratégia Saúde da Família: relato de experiência. Revista Brasileira de Enfermagem, v. 65, n. 4, p. 691-695, jul./ago. 2012. 
LANTYER, A. S. et al. Ansiedade e qualidade de vida entre estudantes universitários ingressantes: avaliação e intervenção. Revista Brasileira de Terapia Comportamental e Cognitiva, v. 18, n. 2, p. 4-19, 2016.

LEÃO, A. M. et al. Prevalência e Fatores Associados à Depressão e Ansiedade entre Estudantes Universitários da Área da Saúde de um Grande Centro Urbano do Nordeste do Brasil. Revista Brasileira de Educação Médica, v. 42, n. 4, p. 55-65, 2018.

MEDEIROS, P. P.; BITTENCOURT, F. O. Fatores associados à Ansiedade em Estudantes de uma Faculdade Particular. Id on Line Revista Multidisciplinar e de Psicologia, v.10, n. 33, jan. 2017. Disponível em: http://idonline.emnuvens.com.br//id. Acesso em: 07 ago. 2020.

MESSETTI, A. V. L. Estatística Descritiva. Londrina: UEL, 2018.

OMS. Organização Mundial da Saúde. Depression and other common mental disordes: global health estimates. Geneva: OMS, 2017.

PETERMANN, F. Hospital anxiety and depression scale, deutsche version (HADS-D). Zeitschrift für Psychiatrie, Psychologie und Psychotherapie, 2015.

SILVA, D. S. D. et al. Depressão e risco de suicídio entre profissionais de Enfermagem: revisão integrativa. Revista da Escola de Enfermagem da USP, v. 49, n. 6, p. 1023-1031, 2015.

SOUSA, I. M. C. et al. Custo-efetividade em práticas integrativas e complementares: diferentes paradigmas. Journal of Management \& Primary Health Care, v. 8, n. 2, p. 343 350, 2017. ISSN 2179-6750.

SOUZA, D. C. et al. Condições emocionais de estudantes universitários: estresse, depressão, ansiedade, solidão e suporte social. 2017. 90 f. Dissertação (Mestrado em Psicologia) - Universidade Federal do Triângulo Mineiro, Uberaba, 2017.

SVOBODA, W. K. et al. A utilização da Terapia Comunitária Integrativa (TCI) em comunidades acadêmicas de duas universidades brasileiras como tecnologia social leve para melhoria das relações interpessoais e organizacionais. Convención Internacional de Salud. Cuba Salud, 2018.

TEROL-CANTERO, M. C.; CABRERA-PERONA, V. Hospital Anxiety and Depression Scale (HADS) review in Spanish Samples. Anales de Psicología, v. 31, n. 2, p. 494, 2015.

WAGNER, G. A. Tratamento de depressão no idoso além do cloridrato de fluoxetina. Rev Saúde Pública, v. 49, n. 20, 2015. Disponível em: http://www.scielo.br/pdf/rsp/v49/pt_00348910-rsp-S0034-89102015049005835.pdf. Acesso em 08 ago. 2020. DOI: https://doi.org/10.1590/S0034-8910.2015049005835

ZIGMOND, A. S.; SNAITH, R. P. A escala de ansiedade e depressão do hospital. Acta psychiatrica scandinavica, v. 67, n. 6, p. 361-370, 1983. 


\section{Como referenciar este artigo}

BOARETTO, J. P.; SILVA, M. Z. da; MARTINS, E. A. P. Ansiedade e depressão na universidade: contribuições da terapia comunitária integrativa. Temas em Educ. e Saúde, Araraquara, v. 16, n. esp. 1, p. 296-310, set., 2020. e-ISSN 2526-3471. DOI: https://doi.org/10.26673/tes.v16iesp.1.14309

Submetido em: 20/05/2020

Revisões requeridas: $30 / 05 / 2020$

Aprovado em: 25/08/2020

Publicado em: 30/09/2020 